Rapid Reviews COVID-19

\title{
Reviews of "Emergence of a novel SARS-CoV-2 strain in Southern California, USA"
}

Takahiko Koyama ${ }^{1}$, François Balloux ${ }^{2}$, Lee W Riley ${ }^{3}$

${ }^{1}$ IBM Research, Computational Biology Center, USA,

${ }^{2}$ UCL: University College London, Genetics, Evolution and Environment, UK,

${ }^{3}$ Division of Infectious Diseases and Vaccinology School of Public Health, University of California Berkeley

Published on: Feb 16, 2021

DOI: $10.1162 / 2 \mathrm{e} 3983 f 5.8 \mathrm{fd} 75134$

License: Creative Commons Attribution 4.0 International License (CC-BY 4.0). 


\section{Statement from the RR:C19 Editorial Office (February 24, 2020)}

RR:C19 solicits reviews to assess cutting-edge research that has not formally undergone peer review before being publicly posted to preprint servers. On February 11 and 15, 2021, RR:C19 published three expert peer reviews of "Emergence of a novel SARS-CoV-2 strain in Southern California, USA." These reviewers found that this research opened up multiple questions that should be further pursued regarding the prevalence and features of the new CAL20C (20C/S452R/B.1.429) variant identified in Southern California, but also highlighted multiple areas of concern with the manuscript. Most notably, two of the reviewers found that the study methods on their own are not conclusive or robust enough to support its claims.

More research has since come out regarding this emerging variant, adding nuance to and concern about the findings presented. Viral mutations are bound to occur in the context of widespread person-to-person transmission, and, as RR:C19 reviewers initially noted, are not in and of themselves necessarily clinically nor epidemiologically relevant. Journalists should exercise caution in reporting the results of the increasing (and important) genomic surveillance conducted throughout the world, as "variants" are entirely inevitable, occur whether or not they are surveilled, and the implications of emerging variants require careful interpretation. Surveillance capacity across the United States and globally is also highly variable, making the situation even more complex.

Additional studies are needed to better understand the roles that variants such as Cal20 have on prevention, policy, and practice. Since RR:C19 published its peer reviews, the areas of concern identified by our reviewers were also addressed by $J \underline{A M A}$ on February 11, 2021, Science on February 23, 2021, andby scientists in many media outlets. In a landscape where emerging Sars-CoV2 variants are increasingly being identified, thus raising concerns to the public, increased surveillance and timely engagement and response by the scientific community are critical to assess the validity and implications of the research.

To read the original manuscript, click the link above.

Summary of Reviews: This preprint, for which additional peer reviews are pending, identifies a CAL2OC variant, which has spread throughout southern California. Additional epidemiological research is needed to determine if this variant could be responsible for increased transmissibility state-wide.

\section{Reviewer 1 (Takahiko Koyama) |}

\section{Reviewer 2 (François Balloux)}

\section{Reviewer 3 (Lee W Riley)}


RR:C19 Strength of Evidence Scale Key.

प्रिप = Misleading

प्र००० = Not Informative

प्र०० = Potentially Informative

प्र०प्र = Reliable

प्राप्र = Strong

To read the reviews, click the links below. 